\title{
Intrinsic Differences in Various Segments of the Proximal Convoluted Tubule
}

\author{
HarRy R. Jacobson and Juha P. KokKo \\ From the Department of Internal Medicine, The University of Texas \\ Southwestern Medical School, Dallas, Texas 75235
}

\begin{abstract}
A в S T R A C T Until recently it has not been possible to compare directly the function of superficial and juxtamedullary nephrons. The present studies, using in vitro microperfusion, were designed to examine whether functional differences exist between proximal convoluted tubule segments of superficial and juxtamedullary nephrons. Electrophysiological studies showed that major differences exist between the relative chloride and sodium permeabilities of these segments. In the $1 \mathrm{st} \mathrm{mm}$ of the superficial proximal convoluted tubule, the permeability to sodium was greater than that to chloride, whereas in the $2 \mathrm{nd} \mathrm{mm}$ of the superficial proximal convoluted tubule and all later segments, the permeability to chloride was greater than that to sodium. The juxtamedullary proximal convoluted tubule was found to differ from the superficial proximal convoluted tubule in two respects: first, the relative permeabilities to chloride and sodium did not differ in the various segments of the juxtamedullary proximal convoluted tubule; second, the permeability to sodium was greater than to chloride throughout. When perfused with a solution lacking glucose and amino acids, the superficial and juxtamedullary convolutions exhibited the same transepithelial potential change, a reversible decrease to less than $-1 \mathrm{mV}$. It thus appears that in both convolutions there exists electrogenic sodium transport coupled to the transport of these organic solutes. This differs from pars recta of both of these nephrons, which have been shown to exhibit electrogenic sodium transport independent of organic solutes. However, when perfused with a solution lacking glucose and amino acids but also containing high chloride and low bicarbonate concentrations, the superficial convolution developed a significantly more positive potential than the juxtamedullary. This difference reflects greater relative chloride permeability in the superficial proximal convolution.
\end{abstract}

Received for publication 10 June 1975 and in revised form 5 December 1975.
These studies show that intrinsic functional differences exist between proximal convoluted tubules obtained from the superficial and juxtamedullary nephron populations.

\section{INTRODUCTION}

It has been recognized for almost 70 years that the mammalian kidney has two major subpopulations of nephrons, based on their location and depth of penetration into the medulla of their loops of Henle (1). Final urine volume and composition depend on the contributions of both these nephron groups, and thus differences in their function may play an important role in what constitutes the final urine. The two nephron populations have been shown to have different filtration rates and different anatomical and functional circulations and differing responses to various changes in physiological parameters such as blood volume (2-6). However, differences between these nephrons can also exist with respect to peritubular environment, intrinsic membrane characteristics, and/or intraluminal fluid constituents. Until recently, experimental observations of renal function have been concerned with the kidney as a whole or with superficial nephrons alone. Only one previous study has directly examined for intrinsic functional differences between nephron segments from superficial and juxtamedullary nephrons. Kawamura et al. (7), using in vitro microperfusion of rabbit nephrons, have recently shown, significant functional differences between straight segments or pars recta obtained from superficial and juxtamedullary nephrons. These investigators have shown differing relative $\mathrm{Na}$ and $\mathrm{Cl}$ permeabilities, different reflection coefficients for $\mathrm{NaCl}$, and different osmotic water permeabilities. In addition to intrinsic membrane differences, differences in function may exist on the basis of compositional changes of the intraluminal fluid as it courses through the tubule. Kokko has shown (8) that in the isolated perfused superficial proximal convoluted tubule, alteration of the composition of the perfusate with respect 
to glucose, amino acids, and sodium bicarbonate significantly affects the transepithelial potential difference (PD). It thus was concluded (8) that the latter segment of the proximal tubule differs from the early segment of the proximal tubule in part by virtue of the intraluminal constituents. The fluid in the early segment is similar to ultrafiltrate of plasma, while the fluid in the latter segment has a high concentration of chloride and a low concentration of glucose, amino acids, and $\mathrm{HCO}_{3}$.

The purpose of the present studies was twofold: first, to evaluate relative permeabilities to $\mathrm{Cl}$ and $\mathrm{Na}$ in the superficial and juxtamedullary proximal convolutions; and second, to examine whether all segments of the proximal convoluted tubule have the capacity of electrogenic sodium transport coupled to organic solute transport.

\section{METHODS}

Segments of proximal convoluted tubules of New Zealand white rabbits were isolated and perfused in vitro by techniques described previously (9). Briefly, female New Zealand white rabbits weighing $1.5-2.5 \mathrm{~kg}$ were fed a standard laboratory diet and had free access to water before guillotine decapitation. The left kidney was quickly removed and cut into 1-2-mm-thick slices. Tubules were dissected from one of these slices in a dish of chilled rabbit serum kept at $\mathrm{pH} 7.4$ by continuous bubbling with a $95 \% \quad \mathrm{O}_{2}$ and $5 \%$ $\mathrm{CO}_{2}$ gas mixture. Seven different proximal convoluted segments were dissected: (a) early superficial proximal convoluted tubule (SFPCT) ${ }^{1}$ less than $1 \mathrm{~mm}$ from its glomerulus; (b) the 2nd mm of the SFPCT; (c) mid SFPCT including segments whose glomerular attachment was not identified and were thus probably more than $1 \mathrm{~mm}$ from the glomerulus; (b) late SFPCT more than $1 \mathrm{~mm}$ from the anatomical pars recta (these were convoluted segments attached to the pars recta but with over $1 \mathrm{~mm}$ of clearly convoluted tubule between the perfusion site and the anatomical or relatively straight pars recta); $(e)$ the 1 st $\mathrm{mm}$ of the juxtamedullary proximal convoluted tubule (JMPCT) ; $(f)$ the mid JMPCT; and $(g)$ the late JMPCT. All segments from the SFPCT were dissected from the most superficial cortex, and all segments from the JMPCT were dissected from immediately above the cortico-medullary junction.

All tubules were perfused in a small Lucite bath containing commercially available rabbit serum kept at $37^{\circ} \mathrm{C}$ and $\mathrm{pH} 7.4$ by continuous bubbling with a $95 \% \mathrm{O}_{2}$ and $5 \%$ $\mathrm{CO}_{2}$ gas mixture. Initial perfusate was either ultrafiltrate of rabbit serum obtained from the same lot as the bathing serum by pressure dialysis with Aminco PM-30 membranes (American Instrument Co., Inc., Silver Spring, Md.) or an artificial solution made to simulate ultrafiltrate. Transepithelial potential differences were measured with the same technique described previously (8), with equivalent bridges of $300 \mathrm{mosm} /$ liter Ringer's in $4 \%$ agarose (PE tubing size 240 ), connected from the bath and perfusion pipet to saturated $\mathrm{KCl}$ containing Beckman (Beckman Instruments, Inc.,

\footnotetext{
${ }^{1}$ Abbreviations used in this paper: $E_{\mathbf{T}}$, transtubular potential; $F$, Faraday's constant; JMPCT, juxtamedullary proximal convoluted tubule; SFPCT, superficial proximal convoluted tubule.
}

Fullerton, Calif.) calomel half-cells. The circuit was completed by placing a voltage reference source and a batteryoperated Keithly model 602 electrometer (Keithly Instruments, Inc., Cleveland, Ohio) in the circuit. This circuit arrangement is completely symmetrical when perfusion and bath solution have the same electrolyte composition. However, the various solutions used in these experiments introduced small liquid junction potentials, which were both calculated and determined experimentally, and then added to or subtracted from the observed PD.

When the bath and perfusion solutions differed only in the concentrations of a single salt, the liquid junction potential, in this case a dilution potential, was calculated from the Nernst-Planck equation as modified by Barry and Diamond (10), with concentrations instead of activities:

$$
E_{L}=-\frac{\mathrm{RT}\left(\mu_{1}-\mu_{3}\right)}{F\left(\mu_{1}-\mu_{3}\right)} \ln \frac{a^{\prime \prime}}{a^{\prime}}
$$

where $\mu$ 's are mobilities, subscript 1 refers to the cation, subscript 3 refers to the anion, $a^{\prime}$ and $a^{\prime \prime}$ are the concentrations of the salt in the two solutions in question, $R$ is the gas constant, $\mathrm{T}$ is absolute temperature, and $F$ is Faraday's constant.

When the bath and perfusate had differing concentrations of two salts with a common ion, a bi-ionic junction potential developed and was calculated from the Nernst-Planck equation as modified by Barry and Diamond (10):

$$
\begin{array}{r}
E_{L}=-\frac{\mathrm{RT} \mu_{1}\left(a^{\prime \prime}{ }_{1}-a_{1}^{\prime}\right)+\mu_{2}\left(a_{2}^{\prime \prime}{ }_{2}-a_{2}^{\prime}\right)-\mu_{3}\left(a^{\prime \prime}{ }_{3}-a_{3}^{\prime}\right)}{F \mu_{1}\left(a^{\prime \prime}{ }_{1}-a_{1}^{\prime}\right)+\mu_{2}\left(a^{\prime \prime}{ }_{2}-a_{2}^{\prime}\right)-\mu_{3}\left(a^{\prime \prime}{ }_{3}-a_{3}^{\prime}\right)} \\
\times \ln \frac{\mu_{1} a^{\prime \prime}{ }_{1}+\mu_{2} a^{\prime \prime}{ }_{2}+\mu_{3} a^{\prime \prime}{ }_{3}}{\mu_{1} a_{1}^{\prime}+\mu_{2} a_{2}+\mu_{3} a_{3}^{\prime}}
\end{array}
$$

where $\mu$ refers to the mobility of each ion, subscripts 1,2 , and 3 refer to the ions themselves (in this case $\mathrm{Cl}, \mathrm{HCO}_{3}$, and $\mathrm{Na}$ ), $a$ is the activity coefficient (in this case the concentration) and " and ' refer to the luminal and bath fluids, respectively. Equivalent conductances at $25^{\circ} \mathrm{C}$ were used instead of mobilities. The limitations and sources of error in these calculations are minimal and have been previously discussed (8).

The liquid junction potentials introduced by the various test solutions were also determined experimentally. Fig. 1 shows a schematic representation of the circuit used in measuring the transtubular potential. Following the circuit diagram below the figure reveals that the liquid junctions from $E_{1}$ through $E_{5}$ are canceled by those from $E_{8}$ through $E_{10}$. Thus, the observed potential is the transtubular potential or $E_{T}$. The first experimental method used to measure the liquid junction potentials is shown in Fig. 2. This circuit is identical to that used for measuring transtubular potentials with two exceptions: no tubule is in place, and the Ringer's agarose bridge between the saturated $\mathrm{KCl}$ and the test tube containing Ringer's bicarbonate in the right-hand side of the figure has been replaced by a saturated $\mathrm{KCl}$ agarose bridge. With perfusate and bath containing Ringer's bicarbonate, the electrometer was centered on zero. Replacing the test tube in the right-hand side of the figure with a test tube containing a solution of different electrolyte composition introduces two liquid junction potentials. The first junction potential between the test solution and the saturated $\mathrm{KCl}$ was assumed to be close to zero. The second junction potential between the test solution and the Ringer's bicarbonate agarose bridge was measured as 

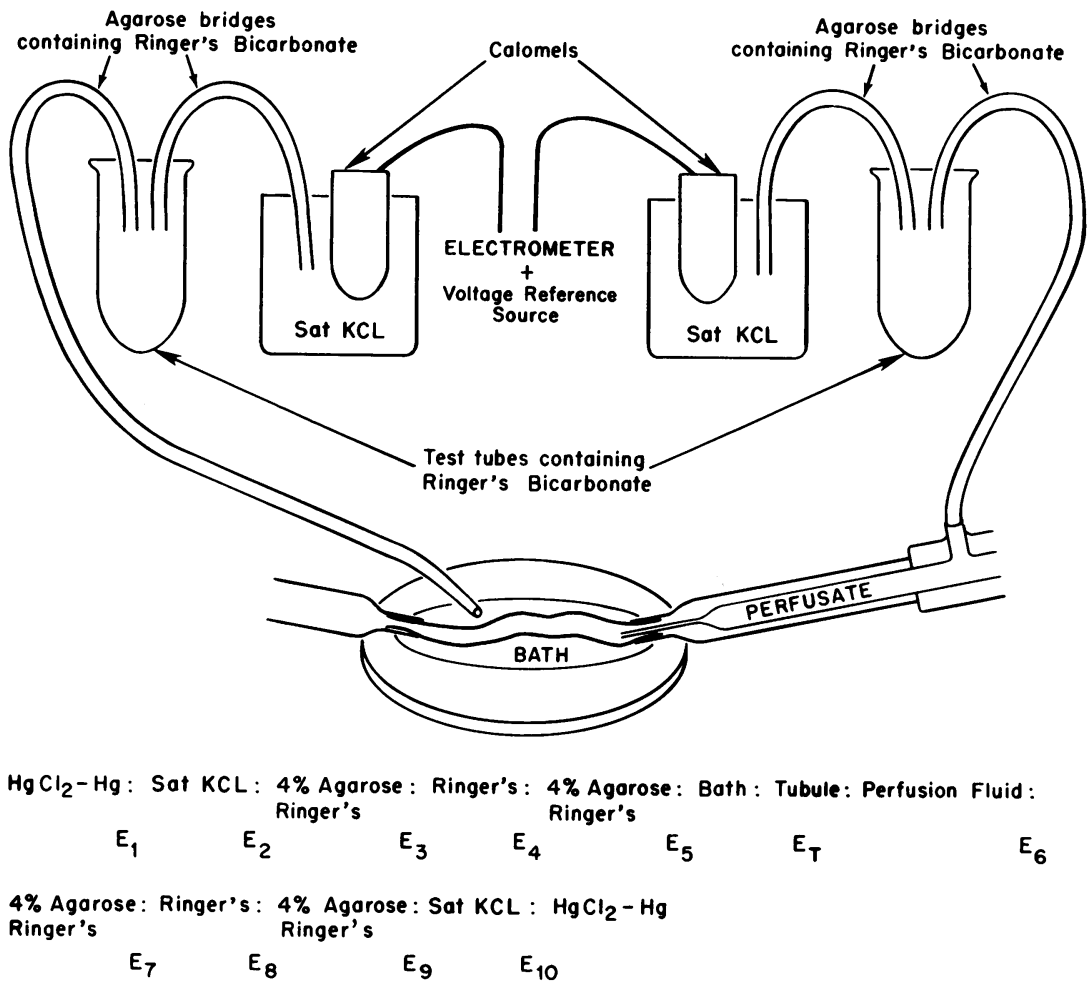

FIGURE 1 Schematic representation of the circuit used to measure transepithelial potentials in isolated tubules.

a deflection from zero on the electrometer. It is interesting to note that the value of the junction potential with A-50 solution (see Table I for composition) was quite close to the calculated value with the modified Nernst-Planck equation, $3.2 \mathrm{mV}$ versus $3.56 \mathrm{mV}$, while that of $B$ solution (see Table I) was not, $0.7 \mathrm{mV}$ versus $2.7 \mathrm{mV}$. The reason for this discrepancy with $B$ solution is unknown. However, to determine if it was related to an effect of agarose on the mobility of $\mathrm{HCO}_{3}^{-}$, a second circuit was utilized as shown below. This circuit was also used to test A-50

TABLE I

Composition of Artificial Solutions

\begin{tabular}{lcccc}
\hline & A & A-50 & B & E \\
\hline & & \multicolumn{3}{c}{$m M$} \\
$\mathrm{NaCl}$ & 105 & 55 & 135 & 105 \\
$\mathrm{KCl}$ & 5 & 5 & 5 & 5 \\
$\mathrm{NaHCO}_{3}$ & 25 & 25 & 5.6 & 25 \\
$\mathrm{CaCl}_{2}$ & 1.8 & 1.8 & 1.8 & 1.8 \\
$\mathrm{MgSO}_{4}$ & 1 & 1 & 1 & 1 \\
$\mathrm{Na}_{2} \mathrm{HPO}_{4}$ & 4 & 4 & 2 & 4 \\
$\mathrm{Na}$ acetate & 10 & 10 & 5 & 10 \\
Glucose & 8.3 & 8.3 & - & - \\
Alanine & 5 & 5 & - & - \\
Mannitol & - & - & 12 & 12 \\
Sucrose & - & 92 & - & - \\
Osmolality of all solutions, & $298-302$. & & \\
\hline
\end{tabular}

solution.

$\mathrm{HgCl}_{2}-\mathrm{Hg}$ : A solution : perfusion fluid : $4 \%$ agarose, $300 \mathrm{mmol} /$ liter Ringer's : $300 \mathrm{mmol} /$ liter $\mathbf{E}_{4}$ Ringer's : $4 \%$ agarose $300 \mathrm{mmol} /$ liter $\mathbf{E}_{5}$

$$
\text { Ringer's : Sat } \mathrm{KCl}: \mathrm{HgCl}_{2}-\mathrm{Hg}
$$

If the two solutions developing $E_{2}$ were identical to each other and had the same electrolyte concentrations as the Ringer's solution, there was no potential in the circuit, and the electrometer was centered at zero. Exchanging the $A$ solution with experimental solutions caused a liquid junction potential to develop at $E_{2}$. With this technique the experimental liquid junction potential for $B$ solution was $1 \mathrm{mV}$ whereas that of A-50 solution was $3.3 \mathrm{mV}$. Therefore, it appears that there is a major discrepancy between the calculated and experimentally determined liquid junction potential with $B$ solution: $0.7 \mathrm{mV}$ and $1 \mathrm{mV}$ experimentally, and $2.7 \mathrm{mV}$ calculated. The experimental and calculated liquid junction potentials for A-50 solution agree closely: $3.2 \mathrm{mV}$ and $3.3 \mathrm{mV}$ experimental and $3.56 \mathrm{mV}$ calculated. The differences in the $\mathrm{B}$ solution junction potentials cannot be due to artifact introduced by agarose.

Because of the discrepancy between the measured and calculated liquid junction potentials with $B$ solution and also because the measurement assumes that a negligible liquid junction potential exists between the saturated $\mathrm{KCl}$ bridge and the test solutions, a further experiment was per- 
formed. The circuit used in this measurement is shown below:

$$
\begin{aligned}
& \mathrm{HgCl}_{2} \text { : bicarbonate : } 4 \% \text { agarose Ringer's } \\
& \mathbf{E}_{\mathbf{\mathbf { E } _ { 2 }}} \\
& \text { bicarbonate : Ringer's bicarbonate : } \mathrm{Ag} / \mathrm{AgCl} \\
& \quad \mathbf{E}_{\mathbf{2}}
\end{aligned}
$$

Although the calomel electrode on the left side of this circuit contains saturated $\mathrm{KCl}$ and thus a liquid junction potential may exist at $E_{1}$, the electrometer was centered on zero to cancel out this possible liquid junction and also the electrode asymetry between the calomel and $\mathrm{Ag} / \mathrm{AgCl}$. Changing the solution between $E_{3}$ and $E_{4}$ with $B$ solution creates a liquid junction potential between $B$ solution and the Ringer's bicarbonate bridge at $E_{3}$. This junction is identical to that occurring during our experiments. The value of this liquid junction potential was measured several times and was found consistently to be between 0.8 and 1.0 $\mathrm{mV}$. It thus appears that the liquid junction potential introduced when our tubules were perfused with B solution was slightly less than $1 \mathrm{mV}$. In accord with this, the results obtained with $B$ solution as perfusate will be shown corrected for both the calculated and measured liquid junction potentials. At present, the reason for the discrepancy between the calculated and measured liquid junctions with B solution is unknown. Whether the liquid junction potential introduced with $B$ solution is in the range of 1 or $2.7 \mathrm{mV}$, the differences between the various tubular segments studied are still the same because all observed potentials were corrected in the same way. However, the previously described attempts at defining the true liquid junction potential are important for a different reason. Earlier studies in membrane physiology have either ignored the presence of liquid junction potentials or used calculated values. Insofar as circumstances may exist where liquid junction potentials differ significantly from theoretical values, an attempt must be made to measure these potentials if interpretation of the data requires accurate knowledge of a true transepithelial potential.

After initiation of perfusion the tubule was allowed to develop a stable PD before perfusate or bath fluids were changed. The composition of the various solutions used is shown in Table I. Solution A was made to stimulate ultrafiltrate of normal serum with respect to electrolyte and organic solute composition. A-50 solution was identical to A solution except for the removal of $50 \mathrm{mmol} /$ liter $\mathrm{NaCl}$, which was isosmotically replaced with sucrose. Solution E was also identical to A solution except for the removal of glucose and alanine, replaced isosmotically with mannitol. Solution B was identical to solution $E$ except for the removal of about $20 \mathrm{mmol}$ of $\mathrm{NaHCO}_{3}$, replaced with $\mathrm{NaCl}$. $\mathrm{B}$ solution was made to simulate in vivo intraluminal fluid in the latter portion of the proximal convolution after the early segment has removed glucose, amino acids, and the majority of the bicarbonate.

An isosmotic $50 \mathrm{mmol} /$ liter $\mathrm{NaCl}$ gradient from lumen to bath was achieved by exchanging the rabbit serum in the bath with A-50 solution. The PD response in the JMPCT, early SFPCT, and mid SFPCT was also observed when A-50 solution was used as perfusate to examine whether the diffusion potential was symmetrical when equivalent salt gradients were imposed from lumen to bath and bath to lumen. Both early and mid SFPCT and JMPCT were examined with $B$ and $E$ solutions as perfusate to determine specifically the role of glucose, amino acid, and bicarbonate removal on the PD. Whenever a segment was perfused with more than one solution it was reperfused with control A solution until its $P D$ returned to its control value. All tubules that did not return to more than $80 \%$ of their control

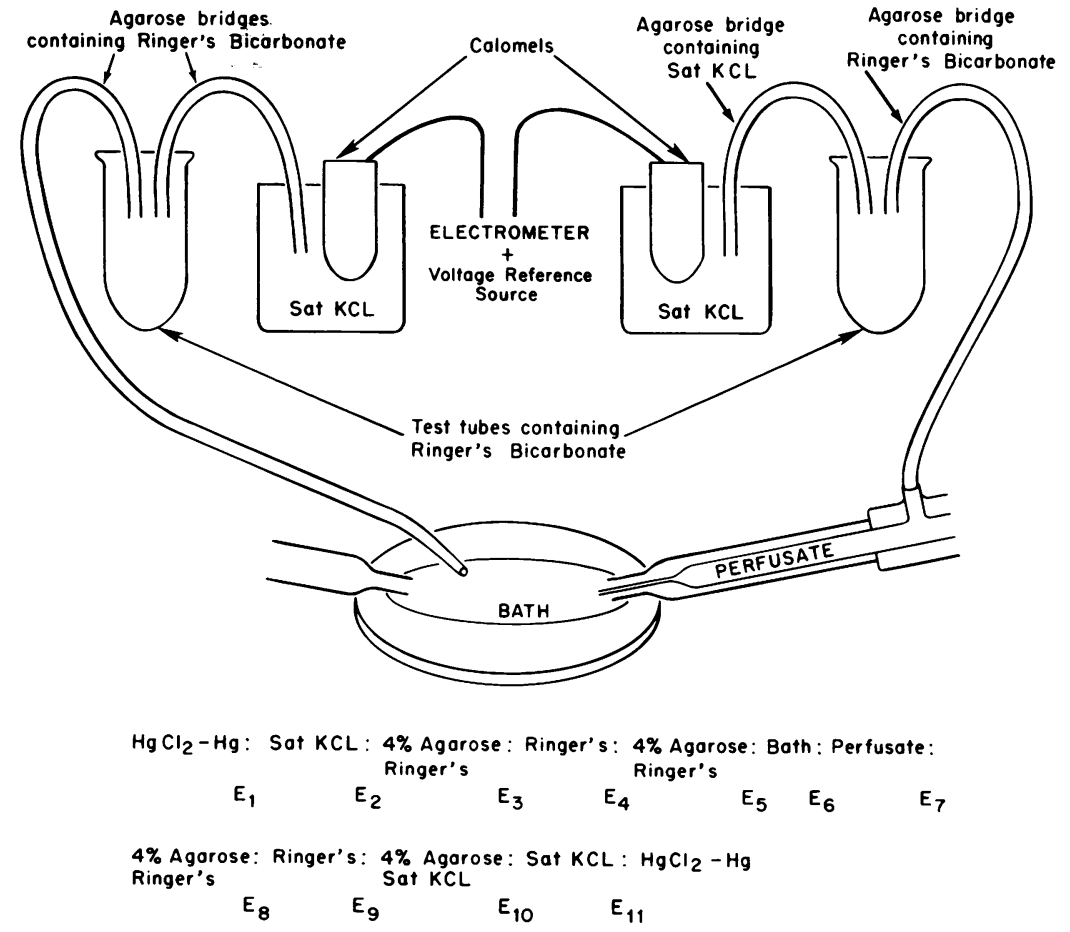

FIGURE 2 Schematic representation of the circuit used to measure liquid junction potentials. 
TABLE II

Transepithelial PD Response to a 50-m $\mathrm{M} \mathrm{NaCl}$ Gradient from Lumen to Bath

\begin{tabular}{lccr}
\hline & $\begin{array}{c}\text { Control } \\
\text { PD* }\end{array}$ & $\begin{array}{c}\text { Experimental } \\
\text { PD } \ddagger\end{array}$ & $n$ \\
\hline & $m V$ & $m V$ & \\
& & & \\
$\begin{array}{l}\text { 1st mm superficial proximal } \\
\quad \text { convolution }\end{array}$ & $-4.3 \pm 0.1$ & $-6.7 \pm 0.3 \S$ & 24 \\
$\begin{array}{l}\text { 2nd mm superficial proximal } \\
\text { convolution }\end{array}$ & $-4.6 \pm 0.3$ & $-1.1 \pm 1.1$ & 5 \\
Mid proximal convolution & $-4.5 \pm 0.2$ & $-0.5 \pm 0.5$ & 25 \\
Late proximal convolution & $-3.1 \pm 0.4$ & $+4.1 \pm 0.5$ & 5 \\
1st mm juxtamedullary & & & \\
$\quad$ convolution & $-4.7 \pm 0.6$ & $-8.7 \pm 1.0$ & 6 \\
Mid juxtamedullary convolution & $-5.1 \pm 0.3$ & $-9.1 \pm 0.6$ & 13 \\
Late juxtamedullary convolution & $-3.8 \pm 0.1$ & $-8.2 \pm 0.4$ & 6 \\
\hline
\end{tabular}

Results are $\pm \mathrm{SE}$.

* Perfusate, A solution; bath, rabbit serum.

† Perfusate, A solution; bath, A-50 solution.

\& All experimental PD's corrected for liquid junction by $+3.5 \mathrm{mV}$.

PD were discarded (less than 1 in 10). All tubules were perfused at more than $10 \mathrm{nl} / \mathrm{min}$, and never in the range where PD has been described to be flow dependent (11). The calculation of the relative permeability ratios of $\mathrm{Cl}$ to $\mathrm{Na}$ was done by the Goldman constant field equation:

$$
\mathrm{PD}=-\frac{\mathrm{RT}}{F} \ln \frac{\mathrm{P}_{\mathrm{Na}}[\mathrm{Na}] l+\mathrm{P}_{\mathrm{Cl}}[\mathrm{Cl}] b}{\mathrm{P}_{\mathrm{Na}}[\mathrm{Na}] b+\mathrm{P}_{\mathrm{Cl}}[\mathrm{Cl}] l}
$$

where $R$ is the gas constant; $\mathrm{T}$ absolute temperature; $F$ the Faraday constant; $P_{\mathrm{Na}}$ and $\mathrm{P}_{\mathrm{O} 1}$ the passive permeability coefficients of $\mathrm{Na}$ and $\mathrm{Cl} ;[\mathrm{Na}]$ and $[\mathrm{Cl}]$ the concentrations of $\mathrm{Na}$ and $\mathrm{Cl}$, and $l$ and $b$ the lumen and bath.

The data for each set of tubules is expressed as the mean $\pm S E M$ of the number of tubules studies. With respect to the $\mathrm{NaCl}$ gradient experiments, statistics were calculated from absolute potential changes. Data with $B$ and $E$ solution as perfusates were analyzed statistically with resultant $\mathrm{PD}$, not $\mathrm{PD}$ changes. Both paired and unpaired $t$ test were utilized when appropriate.

\section{RESULTS}

$P D$ response to removal of $\mathrm{NaCl}$ from bath. Table II shows the $\mathrm{PD}$ response in the various proximal convoluted segments to an isosomotic $50 \mathrm{mmol} /$ liter $\mathrm{NaCl}$ gradient from lumen to bath (A-50 in bath). The early SFPCT (1st $\mathrm{mm}$ ) and all regions of the JMPCT showed negative $\mathrm{PD}$ deflections, indicating greater relative permeability to $\mathrm{Na}$ than $\mathrm{Cl}$ from lumen to bath. The 2nd$\mathrm{mm}$, mid, and late SFPCT exhibited positive PD deflections, indicating greater relative permeability to $\mathrm{Cl}$ than $\mathrm{Na}$ from lumen to bath. The early SFPCT and the entire JMPCT were significantly different from the 2nd-mm, mid, and late SFPCT $(P<0.0005)$. The early SFPCT and JMPCT were not statistically different $(P>0.05)$. The early, mid, and late JMPCT were not different from each other, with all regions exhibiting essentially identical negative $\mathrm{PD}$ deflections, indicating greater relative $\mathrm{Na}$ than $\mathrm{Cl}$ permeability throughout.
Symmetry of the PD response to bidirectional salt gradients. As shown in Table III, when the tubules were exposed alternately to a $50 \mathrm{mmol} /$ liter $\mathrm{NaCl}$ gradient from lumen to bath and bath to lumen (A-50 as bath and as perfusate), the $\mathrm{PD}$ changes were symmetrical. In the early and mid SFPCT there was no statistical difference in the PD changes $(P>0.05)$. In the JMPCT the PD changes were statistically different $(0.5>P>0.025)$, even though the absolute difference in the $\mathrm{PD}$ changes was small ( $3.8 \mathrm{mV}$ PD change with A-50 as bath and 3.3 $m V$ PD change with A-50 as perfusate).

$P D$ response to the removal of glucose, alanine, and $\mathrm{HCO}_{\text {s }}$ from the perfusate. When the early and mid SFPCT and the JMPCT were perfused with solution containing no glucose or alanine (E solution), a significant reversible decrease in the transepithelial PD was observed in all segments (Table IV). All segments exhibited essentially the same response with a reversible decrease in the $P D$ to less than $-1 \mathrm{mV}$ lumen negative. However, with the additional maneuver of $\mathrm{NaHCO}_{3}$ removal and its replacement with $\mathrm{NaCl}$ (B solution), which creates a $\mathrm{Cl}$ concentration gradient from lumen to bath, significant differences were found between the various segments (Table IV). Both the early SFPCT and the JMPCT developed small positive PD's of +0.8 $\mathrm{mV}$ and $+1.2 \mathrm{mV}$, respectively, lumen positive, with a calculated liquid junction potential correction of +2.7 $\mathrm{mV}$. These responses were not statistically different from each other $(P>0.05)$. The mid SFPCT (also with a liquid junction correction of $+2.7 \mathrm{mV}$ ), on the other hand, developed a significantly more positive $\mathrm{PD}$ of $+2.9 \mathrm{mV}$ when compared to the early SFPCT and JMPCT $(P<0.0005)$. These differences are attributed to the different $\mathrm{Cl}$ permeabilities of these segments. The PD's corrected by the experimentally determined liquid junction potential for B solution are also shown in Table IV. Although the differences between the segments are unchanged with a liquid junction correction of $+0.7 \mathrm{mV}$

TABLE III

Symmetrical PD Resłonse to a 50-mM NaCl Gradient from Lumen to Bath and Bath to Lumen

\begin{tabular}{|c|c|c|c|c|}
\hline & $\begin{array}{l}\text { Control } \\
\mathrm{PD} \pm \mathrm{SE}\end{array}$ & $\begin{array}{l}\triangle \mathrm{PD} A-50 \\
\text { Bath } \pm \text { SE\& }\end{array}$ & $\begin{array}{c}\Delta \text { PD A-50 } \\
\text { Perfusate } \pm \text { SE } 8\end{array}$ & $n$ \\
\hline & \multicolumn{2}{|c|}{$m V$} & $m V$ & \\
\hline $\begin{array}{l}\text { 1st mm superficial } \\
\text { convolution }\end{array}$ & $-4.4 \pm 0.2$ & $-2.64 \pm 0.4$ & $+2.86 \pm 0.41^{*}$ & 5 \\
\hline $\begin{array}{l}\text { Mid superficial } \\
\text { convolution }\end{array}$ & $-4.2 \pm 0.1$ & $+4.05 \pm 1.0$ & $-3.71 \pm 1.1^{*}$ & 6 \\
\hline $\begin{array}{l}\text { Juxtamedullary } \\
\text { convolution }\end{array}$ & $-5.0 \pm 0.3$ & $-3.77 \pm 0.4$ & $+3.28 \pm 0.3 \ddagger$ & 7 \\
\hline
\end{tabular}

$* P>0.05$.

$\ddagger 0.05>P>0.025$.

All PD's corrected for liquid junction potential $+3.5 \mathrm{mV}$ with A-50 as bath and $-3.5 \mathrm{mV}$ with A-50 as perfusate. 
TABLE IV

$P D$ Response to E and B Solutions as Perfusate

\begin{tabular}{|c|c|c|c|c|c|c|}
\hline \multirow{4}{*}{$\begin{array}{l}\text { 1st mm superficial convolution } \\
\text { Mid superficial convolution } \\
\text { Juxtamedullary convolution }\end{array}$} & \multirow{4}{*}{$\begin{array}{c}\text { Control } \\
-4.3 \pm 0.3 \\
-4.3 \pm 0.2 \\
-5.3 \pm 0.4\end{array}$} & \multirow{3}{*}{$\begin{array}{c}\begin{array}{c}\text { E solution } \\
\left(\begin{array}{c}\text { s glucose or } \\
\text { amino acids }) \\
(n)\end{array}\right.\end{array} \\
-0.6 \pm 0.2(6) \\
-0.8 \pm 0.1(9)\end{array}$} & \multirow{2}{*}{$\begin{array}{c}\text { Control } \\
-4.3 \pm 0.2\end{array}$} & \multicolumn{3}{|c|}{$\begin{array}{c}\text { B solution } \\
\left(\uparrow \mathrm{Cl} \underset{\left.\downarrow \mathrm{HCO}_{3}\right)}{(n)^{3}}\right.\end{array}$} \\
\hline & & & & $+1.2 \pm 0.2(7)^{*}$ & $-0.8 \ddagger$ & $-0.5 \S$ \\
\hline & & & $-4.3 \pm 0.2$ & $+2.9 \pm 0.1(10)$ & +0.9 & +1.2 \\
\hline & & $-0.6 \pm 0.2(12)$ & $-5.2 \pm 0.4$ & $+0.8 \pm 0.1$ & -1.2 & -0.9 \\
\hline
\end{tabular}

Bath solution was rabbit serum in all experiments. Results are $\pm \mathrm{SE}$.

* With calculated liquid junction potential of $+2.7 \mathrm{mV}$.

$\ddagger$ With experimental liquid junction potential of $+0.7 \mathrm{mV}$.

$\S$ With experimental liquid junction potential of $+1.0 \mathrm{mV}$.

or $+1 \mathrm{mV}$ instead of $+2.7 \mathrm{mV}$, the absolute $\mathrm{PD}$ values changed importantly to $-1.2 \mathrm{mV}$ to $-0.9 \mathrm{mV}$ for the JMPCT, -0.8 to $-0.5 \mathrm{mV}$ for the early SFPCT, and +0.8 to +1.1 for the mid SFPCT (Table IV). Although not depicted in Table IV, when the early and mid SFPCT and JMPCT were perfused with and bathed in $\mathrm{B}$ solution, the PD became zero.

\section{DISCUSSION}

It has been shown previously that the lumen negative transepithelial PD in the superficial proximal convoluted tubule is secondary to active transport processes and not entirely due to diffusion potentials (11). Subsequently Kokko (8) reported that the PD was dependent in a major way on the presence of glucose and amino acids in the luminal fluid. The present studies confirm this concept with respect to the SFPCT and show in addition that the JMPCT also appears to exhibit electrogenic $\mathrm{Na}$ transport coupled to organic solute transport. This is based on the observation that removal of glucose and amino acids from the perfusate always caused a less negative transtubular PD in all segments of the proximal convoluted tubule examined (Table IV). It thus appears that all proximal convoluted segments, superficial and juxtamedullary, regardless of distance from the glomerulus, have the capacity to transport sodium electrogenically when glucose and amino acids are present in the luminal fluid. In contrast to both the SFPCT and JMPCT, the pars recta of superficial and juxtamedullary nephrons

TABLE V

Relative Permeability of $\mathrm{Cl}$ to $\mathrm{Na}$

\begin{tabular}{ll} 
1st mm superficial convolution & 0.64 \\
2nd mm superficial convolution & 1.7 \\
Mid superficial convolution & 2.0 \\
Late superficial convolution & 3.2 \\
Juxtamedullary convolution (all segments) & 0.49 \\
\hline
\end{tabular}

have been shown to exhibit electrogenic $\mathrm{Na}$ transport independent of organic solute transport (8).

It is now widely accepted that electrophysiological techniques can be used to measure relative ion permeabilities. This is the first such attempt to measure the relative $\mathrm{Cl}$-to- $\mathrm{Na}$ permeabilities along the length of the proximal convoluted tubule, and further to compare the SFPCT to the JMPCT. These studies show first that the SFPCT has different intrinsic functional properties along its length with respect to relative $\mathrm{Cl}$ and $\mathrm{Na}$ permeabilities. The relative $\mathrm{Cl}$-to- $\mathrm{Na}$ permeability increases from glomerulus distally, as shown in Table V. Second, since the JMPCT has never been studied before, these experiments not only describe the relative $\mathrm{Cl}$-to- $\mathrm{Na}$ permeabilities in the JMPCT but also show that the JMPCT and SFPCT differ functionally in two major ways: the JMPCT does not appear to have differing Cl-to-Na permeabilities along its length; the JMPCT has a greater relative $\mathrm{Na}$-to- $\mathrm{Cl}$ permeability throughout, while the SFPCT has a greater relative Cl-to- $\mathrm{Na}$ permeability along the majority of its length. Third, the symmetry of the PD change due to equivalent salt gradients imposed on either side of the tubule suggests that the ions involved in generating the diffusion PD permeate through identical pathways from lumen to bath and bath to lumen, rather than through two membranes of differing permeabilities. The symmetry of the bidirectional diffusion PD's also suggest that the PD changes with imposed salt gradients are not secondary to alteration of the baseline active transport. These studies are therefore in agreement with previous electrophysiological studies of the SFPCT in the dog kidney (12), where it is believed that passive permeabilites determined electrophysiologically with salt gradients reflect the permeability of a low-resistance paracellular pathway.

The experiments using a high- $\mathrm{Cl}$, low- $\mathrm{HCO}_{3}$ perfusate (B solution) support the differences in the relative Cl-to$\mathrm{Na}$ permeability of the SFPCT and JMPCT. If the intraluminal fluid in the latter portions of the SFPCT and 


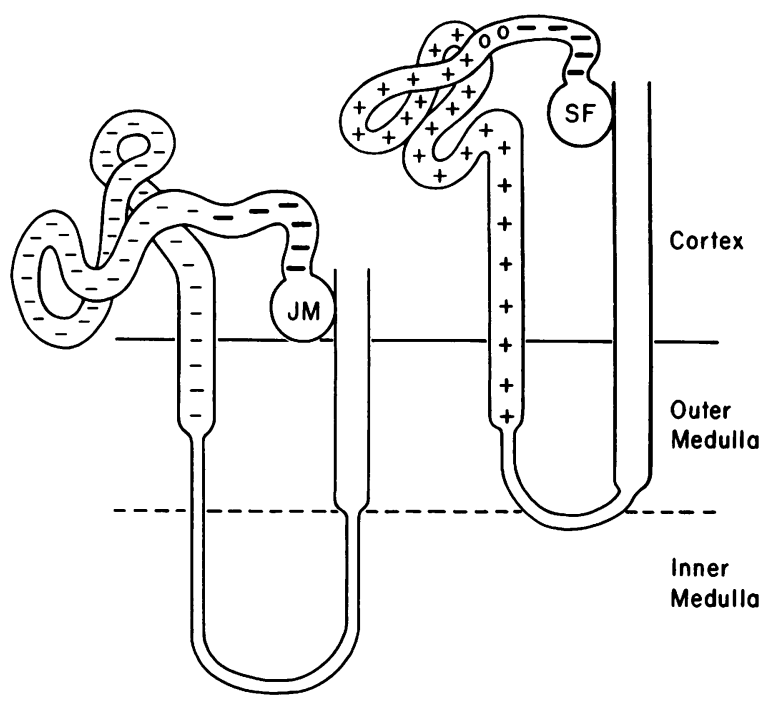

FIGURE 3 Proposed potential profile of the two populations of proximal tubules of the rabbit: superficial (SF) and juxtamedullary (JM). The pars recta data are from ref 7 .

JMPCT in vivo are similar, one would expect differences in the in vivo PD's. These PD differences could be significant because of their resultant differing effects on passive transport phenomena. A positive intraluminal PD would act as a driving force for efflux of $\mathrm{Na}^{+}$, while a negative intraluminal PD would retard the efflux of $\mathrm{Na}^{+}$. Direct analysis of this question will require flux measurements in both the SF- and JMPCT with B solution. At present, calculation of the degree of importance of the chloride diffusion potential (or lack of it) in the SFPCT and JMPCT is difficult because the magnitude of the PD is unclear and the chloride permeability coeffcients in these segments have not been measured. Barret et al. (14) have shown that in the SFPCT of the rat the first loop accessable to micropuncture has a negative $\mathrm{PD}$, while the segments beyond have a positive PD of approximately $+1 \mathrm{mV}$. Kokko (8), using the calculated liquid junction potential correction of $+2.7 \mathrm{mV}$, has previously suggested that the latter portion of the SFPCT has a PD of approximately $+3 \mathrm{mV}$ lumen positive. This previously published $+3 \mathrm{mV} P D$ for the latter portion of the SFPCT is probably too high in view of the discrepancies between the previously calculated and the present directly measured liquid junction potentials (Table IV). With the high-chloride perfusate (B solution) and the directly measured liquid junction PD (in contrast to the calculated value), the PD in the latter segments of the SFPCT is in close agreement with the $\mathrm{PD}$ reported in vivo by Barrett et al. (13).

In summary, the present studies are the first to establish that the SFPCT and JMPCT have intrinsically different characteristics. In the SFPCT the relative Na-to-
$\mathrm{Cl}$ permeability varies along the length of the tubule, whereas in the JMPCT the relative $\mathrm{Na}$-to- $\mathrm{Cl}$ permeability remains constant along the entire length of the tubule. In the SFPCT, $\mathrm{Na}$ is more permeant than $\mathrm{Cl}$ in the 1 st $\mathrm{mm}$ from the glomerulus, whereas in its latter portions $\mathrm{Cl}$ is more permeant than $\mathrm{Na}$. On the other hand, $\mathrm{Na}$ is more permeant than $\mathrm{Cl}$ throughout the JMPCT. These findings suggest that differing diffusion potentials exist along the length of these two populations of nephrons (Fig. 3). We have also shown that the JMPCT is similar to the SFPCT in that it is capable of electrogenic $\mathrm{Na}$ transport coupled to organic solute transport, and, therefore, the PD would be negative in early segment of each nephron when glucose and amino acids exist in significant concentrations (Fig. 3). Since the final urine is the composite of all nephron populations and since in various salt-retaining or -excreting models there exists redistribution of filtrate and blood between superficial and juxtamedullary regions of the cortex, it may be argued that the differing characteristics between the superficial and juxtamedullary nephrons contribute differently to final urine composition.

\section{ACKNOWLEDGMENTS}

The authors would like to express their appreciation to Mrs. Virginia Borcoman and Miss Jan Hawkins for their technical assistance.

This work was supported in part by U. S. Public Health Service Program Grant PO1 HL 11662 and National Institute of Arthritis and Metabolic Diseases Research Grant 1 RO1 AM 14677.

\section{REFERENCES}

1. Huber, G. C. 1909-1910. The morphology and structure of the mammalian renal tubule. Harvey Lect. 5: 100-149.

2. Bartel, A. G., R. M. Zakheim, L. G. Jones, and A. C. Barger. 1967. Redistribution of renal blood flow produced by furosemide and ethacrynic acid. Clin. Res. 21 : 869-878.

3. Jamison, R. L. 1970. Micropuncture study of superficial and juxtamedullary nephrons in the rat. Am. J. Physiol. 218: 46-55.

4. Horster, M., and K. Thurau. 1968. Micropuncture studies on the filtration rate of single superficial and juxtamedullary glomeruli in the rat kidney. Pfliggers Archiv. Gesamte Physiol. Menschen Tiere. 301: 162.

5. Hollenberg, N. K., M. Epstein, R. D. Guttmann, M. Conroy, R. T. Basch, and J. P. Merrill. 1970. Effect of $\mathrm{Na}$ balance on intrarenal distribution of blood flow in normal man. J. Appl. Physiol. 28: 312-317.

6. Trueta, J., A. E. Barclay, P. M. Daniel, K. J. Franklin, and M. M. L. Prichard. 1947. Studies of the Renal Circulation. Blackwell Scientific Publications Ltd., Oxford, England. 187 pp.

7. Kawamura, S., M. Imai, D. W. Seldin, and J. P. Kokko. 1975. Characteristics of salt and water transport in superficial and juxtamedullary straight segments of proximal tubules. J. Clin. Invest. 55 : 1269-1277. 
8. Kokko, J. P. 1973. Proximal tubule potential difference. Dependence on glucose, $\mathrm{HCO}_{2}$, and amino acids. J. Clin. Invest. 52 : 1362-1367.

9. Burg, M., J. Grantham, M. Abramow, and J. Orloff. 1966. Preparation and study of fragments of single rabbit nephrons. Am. J. Physiol. 210: 1293-1298.

10. Barry, P. H., and J. M. Diamond. 1970. Junction potentials, electrode standard potentials and other problems in interpreting electrical properties of membranes. J. Membr. Biol. 3: 93-122.
11. Kokko, J. P., and F. C. Rector. 1971. Flow dependence of transtubular potential difference in isolated perfused segments of rabbit proximal convoluted tubule. J. Clin. Invest. 50: 2745-2750.

12. Boulpaep, E. L., and J. F. Seely. 1971. Electrophysiology of proximal and distal tubules in the autoperfused dog kidney. Am. J. Physiol. 221 : 1084-1096.

13. Barratt, L. J., F. C. Rector, J. P. Kokko, and D. W. Seldin. 1974. Factors governing the transepithelial potential difference across the proximal tubule of the rat kidney. J. Clin. Invest. 53: 454-464. 